\title{
Policy
}

\section{Best Practices in Videoconferencing-Based Telemental Health April 2018}

Jay H. Shore, $M D, M P H_{1}^{1,2}$ Peter Yellowlees $M D, M B B S^{3}$

Robert Caudill, $M D_{1}^{4}$ Barbara Johnston, MSN, ${ }^{5}$

Carolyn Turvey, PhD, ${ }^{6}$ Matthew Mishkind, PhD, ${ }^{7}$

Elizabeth Krupinski, $P h D_{1}^{7}$ Kathleen Myers, $M D, M P H_{1}^{8}$

Peter Shore, PsyD, ${ }^{9}$ Edward Kaftarian, MD, ${ }^{10}$ and Donald Hilty, MD ${ }^{11}$

${ }^{1}$ Helen and Arthur E. Johnson Depression Center, the University

of Colorado Anschutz Medical Campus, Aurora, Colorado.

${ }^{2}$ Department of Psychiatry, the University of Colorado

Anschutz Medical Campus, Aurora, Colorado.

${ }^{3}$ Department of Psychiatry, University of California, Davis,

Sacramento, California.

${ }^{4}$ Department of Psychiatry, The University of Louisville

School of Medicine, Louisville, Kentucky.

${ }^{5}$ HealthLinkNow, Sacramento, California.

${ }^{6}$ Department of Psychiatry, Carver College of Medicine,

The University of lowa, lowa City, lowa.

${ }^{7}$ Department of Radiology and Imaging Sciences, Emory

University School of Medicine, Atlanta, Georgia.

${ }^{8}$ Center for Child Health, Behavior, and Development,

Seattle Children's Hospital, Seattle, Washington.

${ }^{9}$ Portland Veterans Affairs Health Care System, Portland, Oregon.

${ }^{10}$ Orbit Health Telepsychiatry, Encino, California.

${ }^{11}$ Northern California Veterans Affairs Health Care System,

Sacramento, California.

\section{Abstract}

Telemental health, in the form of interactive videoconferencing, has become a critical tool in the delivery of mental health care. It has demonstrated the ability to increase access to and quality of care, and in some settings to do so more effectively than treatment delivered in-person. This article updates and consolidates previous guidance developed by The American Telemedicine Association (ATA) and The American Psychiatric Association (APA) on the development, implementation, administration, and provision of telemental health services. The guidance included in this article is intended to assist in the development and delivery of effective and safe telemental health services founded on expert consensus, research evidence, available resources, and patient needs. It is recommended that the material reviewed be contemplated in conjunction with APA and ATA resources, as well as the pertinent literature, for additional details on the topics covered.

Keywords: telemedicine, telehealth, telemental health, policy

\section{Introduction}

his document represents a collaboration between the American Psychiatric Association (APA) and the American Telemedicine Association (ATA) to create a consolidated update of the previous APA and ATA official documents and resources in telemental health to provide a single guide on best practices in clinical videoconferencing in mental health. The APA is the main professional organization of psychiatrists and trainee psychiatrists in the United States, and the largest psychiatric organization in the world. The ATA, with members from throughout the United States and the world, is the principal organization bringing together telemedicine practitioners, health care institutions, government agencies, vendors, and others involved in providing remote health care using telecommunications.

Telemental health in the form of interactive videoconferencing has become a critical tool in the delivery of mental health care. It has demonstrated its ability to increase access and quality of care, and in some settings to do so more effectively than treatment delivered in-person.

The APA and the ATA have recognized the importance of telemental health with each individual association undertaking efforts to educate and provide guidance to their members in the development, implementation, administration, and provision of telemental health services. It is recommended that this guide be read in conjunction with the other APA and ATA resources that provide more detail. ${ }^{1-7}$

\section{OFFICIAL APA AND ATA GUIDELINES, RESOURCES,} AND TELEMENTAL HEALTH TRAININGS APA

(1) APA Web-based Telepsychiatry Toolkit (2016) ${ }^{1}$

(2) Resource Document on Telepsychiatry and Related Technologies in Clinical Psychiatry, Council on Law and Psychiatry (2014) ${ }^{8}$

(3) American Psychiatric Association. Telepsychiatry via Videoconferencing. (1998) ${ }^{3}$

\section{ATA}

(4) Practice Guidelines for Telemental Health with Children and Adolescents (2017)

(5) Telemental Health Resource Toolbox (2017) ${ }^{9}$

(6) Delivering Online Video Based Mental Health Services $(2014)^{5}$

(7) A Lexicon of Assessment and Outcome Measures for Telemental Health (2013) ${ }^{10}$

(8) Practice Guidelines for Video-Based Online Mental Health Service $(2013)^{6}$

(9) Practice Guidelines for VideoconferencingBased Telemental Health (2009) ${ }^{7}$

(10) Evidence-Based Practice for Telemental Health $(2009)^{11}$ 


\section{SHORE ET AL.}

These guidelines focus on interactive videoconferencingbased mental health services (a.k.a., telemental health). The use of other technologies such as virtual reality, electronic mail, electronic health records, telephony, remote monitoring devices, chat rooms, or social networks is not a focus of this document except where these technologies interface with videoconferencing services.

The document was created by a joint writing committee drawn from the APA Committee on Telepsychiatry and the ATA Telemental Health Special Interest Group (TMH SIG). This document draws directly from ATA's three previous guidelines, selecting from key statements/guidelines, consolidating them across documents, and then updating them where indicated. Following internal review processes within the APA and the ATA, the Board of Directors of the ATA and the Joint Reference Committee (JRC) of the APA have given approval to its publication.

The reference list includes several detailed reviews providing justification and documentation of the scientific evidence supporting telemental health. ${ }^{12-16}$ Following ATA guideline writing convention, this document contains requirements, recommendations, or actions that are identified by text containing the keywords "shall," "should," or "may." "Shall" indicates that it is required whenever feasible and practical under local conditions. "Should" indicates an optimal recommended action that is particularly suitable, without mentioning or excluding others. "May" indicates additional points that may be considered to further optimize the telemental health care process.

It should be recognized that compliance with these recommendations will not guarantee accurate diagnoses or successful outcomes. The purpose of this guide is to assist providers in providing effective and safe medical care founded on expert consensus, research evidence, available resources, and patient needs.

This document is not meant to establish a legal standardof-care.

\section{Administrative Considerations PROGRAM DEVELOPMENT}

Providers or organizations delivering mental health services should conduct a telehealth needs assessment before initiating services. This needs assessment should include, at a minimum, the following components: program overview statement, services to be delivered, proposed patient population, provider resources, technology needs, staffing needs, quality and safety protocols, business and regulatory processes, space requirements, training needs, evaluation plan, and sustainability.

\section{LEGAL AND REGULATORY ISSUES}

Licensure and malpractice. Health care services have been defined as delivered in the state where the patient is located. Providers of telemental health services shall comply with state licensure laws, which typically entail holding an active professional license issued by the state in which the patient is physically located during a telemental health session, and shall have appropriate malpractice coverage. Providers shall conduct their own due diligence to determine the type of licensure required, and ensure they are in compliance with state licensing board regulations. If providing care within a federal health care system (e.g., Department of Veterans Affairs, Department of Defense, and Indian Health Service), providers shall follow the specific organization guidelines around licensure, which may allow for a single state licensure across multiple jurisdictions. Providers may utilize the interstate licensure compact or special telemedicine licensures offered by certain states provided they comply with all individual state licensure and program requirements.

Scope of practice. Providers or organizations offering telemental health services shall ensure that the standard-of-care delivered through telemedicine is equivalent to in-person care. Persons engaged in telemental health services shall be aware of their professional organization's positions on telemental health and incorporate the professional association standards and clinical practice guidelines whenever possible. Providers in practice and trainees should stay current with evolving technologies, telemental health research findings, and policies.

Prescribing. Providers shall be aware of both federal and state guidelines around the prescription of controlled substances, including the Ryan Haight Online Pharmacy Consumer Protection Act of 2008. Providers shall comply with federal and state regulations around the prescription of controlled substances based on the setting, model of care, scope of practice, and locations in which they are practicing and where the patient is located at the time of treatment.

Informed consent. Local, state, and national laws regarding verbal or written consent shall be followed. If written consent is required, then electronic signatures, assuming these are allowed in the relevant jurisdiction, may be used. The provider shall document the provision of consent in the medical record.

Billing and reimbursement. The patient shall be made aware of any and all financial charges that may arise from the services to be provided before the commencement of initial services. Appropriate documentation and coding should be undertaken specifying when services are rendered through telemental health. 


\section{BEST PRACTICES IN TELEMENTAL HEALTH}

\section{STANDARD OPERATING PROCEDURES/PROTOCOLS}

Before initiating telemental health services, any organization or provider shall have in place a set of Standard Operating Procedures or Protocols that should include (but are not limited to) the following administrative, clinical, and technical specifications:

- Roles, responsibilities (i.e., daytime and after-hours coverage), communication, and procedures around emergency issues.

- Agreements to assure licensing, credentialing, training, and authentication of practitioners as well as identity authentication of patients according to local, state, and national requirements.

- A systematic quality improvement and performance management process that complies with any organizational, regulatory, or accrediting requirements for outcomes management.

Patient-provider identification. All persons at both sites of the videoconference shall be identified to all participants at the beginning of a telemental health session. Permission from the patient should not be required if safety concerns mandate the presence of another individual or if the patient is being legally detained.

At the beginning of a video-based mental health treatment with a patient, the following information shall be verified and documented:

- The name and credentials of the provider and the name of the patient.

- The location(s) of the patient during the session.

- Immediate contact information for both provider and patient (phone, text message, or e-mail), and contact information for other relevant support people, both professional and family.

- Expectations about contact between sessions shall be discussed and verified with the patient, including a discussion of emergency management between sessions.

\section{Emergencies}

GENERAL CONSIDERATIONS. Professionals shall maintain both technical and clinical competence in the management of mental health emergencies. Provisions for management of mental health emergencies shall be included in any telemental health procedure or protocol. Clinicians shall be familiar with local civil commitment regulations and should have arrangements to work with local staff to initiate/assist with civil commitments or other emergencies.

CLINICALLY SUPERVISED SETTINGS. Clinically supervised settings are patient locations where other medical or support staff are available in real time to support the telemental health sessions. Emergency protocols shall be created with clear explanation of roles and responsibilities in emergency situations. These include determination of outside clinic hours emergency coverage and guidelines for determining when other staff and resources should be brought in to help manage emergency situations. Clinicians shall be aware of safety issues with patients displaying strong affective or behavioral states upon conclusion of a session and how patients may then interact with remote site staff.

CLINICALLY UNSUPERVISED SETTINGS. In instances wherein the mental health provider is providing services to patients in settings without clinical staff immediately available:

- Providers should discuss the importance of having consistency in where the patient is located for sessions and knowing a patient's location at the time of care, as it impacts emergency management and local available resources.

- As patients change locations, providers shall be aware of the impact of location on emergency management protocols. These include emergency regulations, resources (e.g., police, emergency rooms, and crisis teams), and contacts. These should be documented and available to providers.

- For treatment occurring in a setting where the patient is seen without access to clinical staff, the provider should consider the use of a "Patient Support Person" (PSP) as clinically indicated. A PSP is a family, friend, or community member selected by the patient who could be called upon for support in the case of an emergency. The provider may contact the PSP to request assistance in evaluating the nature of emergency and/or initiating 91-1 from the patient's home.

- If a patient and/or a PSP will not cooperate in his or her own emergency management, providers shall be prepared to work with local emergency personnel in case the patient needs emergency services and/or involuntary hospitalization.

Care coordination. With consent from the patient and in accordance with privacy guidelines, telemental health providers should arrange for appropriate and regular communication with other professionals and organizations involved in the care of the patient.

\section{Technical Considerations}

\section{VIDEOCONFERENCING PLATFORM REQUIREMENTS}

Providers and organizations should select videoconferencing applications that have the appropriate verification, confidentiality, and security parameters necessary to be 


\section{SHORE ET AL.}

properly utilized for this purpose. In the event of a technology breakdown, causing a disruption of the session, the professional shall have a backup plan in place (e.g., telephone access). Telemental health shall provide services at a bandwidth and with sufficient resolutions to ensure that the quality of the image and/or audio received is appropriate to the services being delivered.

\section{INTEGRATION OF VIDEOCONFERENCING INTO OTHER TECHNOLOGY AND SYSTEMS}

Organizations shall ensure the technical readiness of the telehealth equipment and the clinical environment. They shall have policies and procedures in place to ensure the physical security of telehealth equipment and the electronic security of data. Organizations shall ensure compliance with all relevant safety laws, regulations, and codes for technology and technical safety.

Privacy, security, and Health Insurance Portability and Accountability Act. For telemental health services provided within the United States, the U.S. Health Insurance Portability and Accountability Act (HIPAA) of 1996, and state privacy requirements, shall be followed at all times to protect patient privacy. Privacy requirements in other countries shall be followed for telemental health services provided in those countries.

Patients receiving mental health and substance use disorder services are afforded a higher degree of patients' rights as well as organizational responsibilities (e.g., need for specific consent from patients to release information around substance use). Telemental health organizations shall be aware of these additional responsibilities and ensure that they are achieved. Telemental health organizations and providers shall determine processes for documentation, storage, and retrieval of telemental health records.

\section{PHYSICAL LOCATION/ROOM REQUIREMENTS}

During a telemental health session, both locations shall be considered a patient examination room regardless of a room's intended use. Providers shall ensure privacy so clinical discussion cannot be overheard by others outside of the room where the service is provided. To the extent possible, the patient and provider cameras should be placed at the same elevation as the eyes with the face clearly visible to the other person. The features of the physical environment for both shall be adjusted so the physical space, to the degree possible, maximizes lighting, comfort, and ambiance.

When asynchronous telemental health consultations are occurring, the interviewer should be appropriately trained, and the digital recording of the interview shall be shared and stored in accordance with HIPAA regulations.

\section{Clinical Considerations}

\section{PATIENT AND SETTING SELECTION}

There are no absolute contraindications to patients being assessed or treated using telemental health. The use of telemental health with any individual patient is at the discretion of the provider. For clinically unsupervised settings (e.g., home and office) where support staff are not immediately available, providers shall consider appropriateness of fit for an individual patient. Provision of telemental health services in professionally unsupervised settings requires that the patient take a more active and cooperative role in the treatment process than would be the case for in-person locales. Patients need to be able to set up the videoconferencing system, maintain the appropriate computer/device settings, establish a private space, and cooperate for effective safety management. Factors to consider include:

- Providers should consider such things as patient's cognitive capacity, history regarding cooperativeness with treatment professionals, current and past difficulties with substance abuse, and history of violence or self-injurious behavior.

- Providers shall consider geographic distance to the nearest emergency medical facility, efficacy of patient's support system, and current medical status.

- The consent process shall include discussion of circumstances around session management so that if a patient can no longer be safely managed through distance technology, the patient is aware that services may be discontinued.

- Providers should consider whether there are any medical aspects of care that would require in-person examination including physical examinations. If the provider cannot manage the medical aspects for the patient without being able to conduct initial or recurrent physical examinations, this shall be documented in the record, and arrangements shall be made to perform physical examinations onsite as clinically indicated.

\section{MANAGEMENT OF HYBRID PATIENT-PROVIDER RELATIONSHIPS}

Telemental health interviews can be conducted as part of a wider in-person and online clinical relationship using multiple technologies by providers working individually or in teams. The telemental health interview can be an adjunct to periodic face-to-face in-person contact or can be the only contact. It is typically supported by additional communication technologies such as faxed or e-mailed consultation information, patient portals, telephone, mobile devices, and electronic health records. Providers should have clear policies pertaining to communications with patients. These should 


\section{BEST PRACTICES IN TELEMENTAL HEALTH}

describe the boundaries around ways in which patients can communicate with a provider, the content of which is appropriate to share over different technology platforms, anticipated response times, and how and when to contact a provider. Providers should identify clearly which platforms are acceptable for communication of an emergency and expected response times. Providers should be attentive of the impact of different technology platforms on patient rapport and communication. All modes of communication of personal health history shall be HIPAA compliant.

\section{ETHICAL CONSIDERATIONS}

Health professionals shall be responsible for maintaining the same level of professional and ethical discipline and clinical practice principles and guidelines as in person care in the delivery of care in telemental health, as well as additional telemental health-related concerns such as consent processes, patient autonomy, and privacy.

\section{CULTURAL ISSUES}

Telemental health providers should be culturally competent to deliver services to the populations that they serve. Providers should familiarize themselves with the cultures and environment where they are working and may use site visits and cultural facilitators to enhance their local knowledge when appropriate and practical. Providers should assess a patient's previous exposure, experience, and comfort with technology/ videoconferencing. They shall be aware of how this might impact initial telemental health interactions. Providers should conduct ongoing assessment of the patient's level of comfort with technology over the course of treatment.

\section{SPECIFIC POPULATIONS AND SETTINGS}

Child/adolescent populations. Telemental health procedures for the evaluation and treatment of youth shall follow the same guidelines presented for adults with modifications to consider the developmental status of youth such as motor functioning, speech and language capabilities, relatedness, and relevant regulatory issues. When working with younger children, the environment should facilitate the assessment by providing an adequate room size, furniture arrangement, toys, and activities that allow the youth to engage with the accompanying parent, presenter, and provider, and demonstrate age-appropriate skills.

Extended participation of family members or other relevant adults is typical of mental health treatment of children and adolescents. Providers should adhere to usual in-person practices for including relevant adults with appropriate modifications for delivering service through videoconfer- encing in the context of resources at the patient site. Extended participation may include a "presenter" who may facilitate sessions (e.g., vital signs, assistance with rating scales, managing active children, and assisting with any urgent interventions). Providers should consider how the presenter's involvement can affect service delivery (e.g., social familiarity with the family, perceived confidentiality, and sharing information with other team members).

When telemental services are delivered outside of traditional clinic settings (e.g., schools), providers should work with staff to ensure safety, privacy, appropriate setting, and accommodations. This is particularly true if multiple staff participate in sessions. Appropriateness for telemental care shall consider safety of the youth, the availability of supportive adults, the mental health status of those adults, and ability of the site to respond to any urgent or emergent situations.

Forensic and correctional. Providers shall be aware of system issues in working in forensic and correctional settings and follow applicable standard consent around both treatment and evaluation in terms of patient's legal status and rights. Providers shall have clear site-specific protocols about working with patients and staff in forensic and correctional settings.

Geriatric. The geriatric patient often has multiple medical problems and the inclusion of family members should be undertaken as clinically appropriate and with the permission of the patient. Interviewing techniques shall be adapted for patients who may be cognitively impaired, find it difficult to adapt to the technology, or have visual or auditory impairment. Cognitive testing may be provided through videoconferencing but might need to be modified for use through video. Organizations administrating cognitive testing through videoconferencing shall be aware of the properties of the individual test instrument, how it may be impacted by videoconferencing, and any potentially needed modifications.

Military, veteran, and other federal populations. Providers shall be familiar with the federal and specific organizational structures and guidelines for patients related to the location of care. Providers should familiarize themselves with the culture of the patients (e.g., military cultural competency) and the organizational systems in which they practice.

Substance use disorder treatment. Providers shall be aware of and comply with federal, state, and local regulations around prescription of controlled substances involved in substance use disorder treatment. Providers shall coordinate with onsite staff to provide appropriate standard of care, including care 


\section{SHORE ET AL.}

coordination and monitoring of physiological parameters for monitoring of ongoing treatment as clinically indicated.

Inpatient and residential settings. Providers should work to integrate themselves into inpatient and residential care settings where they practice through virtual participation in administration and organizational meetings, including clinical case staffing on a routine/regular basis. Remote providers should optimize use of patient site staff for help with telemental health consultations and case coordination as clinically indicated. Inpatient units should provide the telemental health provider with adequate access to patients, members of the interdisciplinary treatment team, and primary medical providers and nursing support when appropriate.

Primary care settings. Providers should be aware of best practice in leveraging telepsychiatry to support integrated care across a continuum of models, including direct patient assessment, consultative models, (e.g., asynchronous), and team-based models of care. Providers practicing integrated care telepsychiatry should attend to the impact of virtual interactions on team processes, dynamics, and patient outcomes in the delivery of integrated care.

Rural. Providers should be familiar with the impact of rural environments on treatment including firearm ownership, kinship in small communities, local geographic barriers to care, and general availability of health care resources.

\section{Acknowledgments}

The authors thank the staff of the ATA and the APA (Sabrina L. Smith, Dr. HA [ATA], Harry Mellon [ATA], Nathan Tatro, MA [APA], and Michelle Dirst, MA [APA]) for their support in preparing this document. The authors also recognize the work of the following reviewers: TMH SIG (ATA), APA Committee on Telepsychiatry (APA), ATA Standards and Guidelines Committee Member (SG), ATA staff (ATAS), and APA staff (APAS). These best practices for videoconferencingbased telemental health have been prepared in collaboration and endorsement by the APA and the ATA. As such, they are copyrighted by the APA.

\section{Disclosure Statement}

No competing financial interests exist.

\section{REFERENCES}

1. APA Telepsychiatry Toolkit. 2016. Available at www.psychiatry.org/ psychiatrists/practice/telepsychiatry (last accessed September 12, 2018).

2. Recupero P, Fisher JCE. APA resource document on telepsychiatry and related technologies in clinical psychiatry. 2014. Available at www.psychiatry.org/
File\%20Library/Psychiatrists/Directories/Library-and-Archive/resource documents/Resource-2014-Telepsychiatry-Clinical-Psychiatry.pdf (last accessed September 12, 2018).

3. American Psychiatric Association. Telemental health via videoconferencing. 1998

4. Myers K, Nelson EL, Rabinowitz T, Hilty D, Baker D, Barnwell SS, Comer JS. American Telemedicine Association practice guidelines for telemental health with children and adolescents. Telemed J E Health 2017;23:779-804.

5. Turvey $C$, Yellowlees $P$, Shore JH, Shore P. Delivering online video based mental health services. American Telemedicine Association Learning Center, 2014. Available at http://learn.americantelemed.org/diweb/catalog/item/id/241193/q/ $0=n \% 26 c=96 \% 26 t=3359$ (last accessed September 12, 2018).

6. Turvey $C_{1}$ Coleman $M$, Dennison 0 , Drude $K$, Goldenson $M$, Hirsch $P_{1}$ Jueneman $R_{1}$ Kramer GM, Luxton DD, Maheu MM, Malik TS, Mishkind MC, Rabinowitz T, Roberts $\sqcup$, Sheeran T, Shore JH, Shore P, van Heeswyk F, Wregglesworth B, Yellowlees $P$, Zucker ML, Krupinski EA, Bernard J. ATA practice guidelines for video-based online mental health services. Telemed J E Health 2013;19:722-730.

7. Yellowlees P, Shore J, Roberts L. Practice guidelines for videoconferencing-based telemental health-October 2009. Telemed J E Health 2010;16:1074-1089.

8. American Psychiatric Association, Resource document on telepsychiatry and related technologies in clinical psychiatry, 2014. Available at https://www .psychiatry.org/File\%20Library/Psychiatrists/Directories/Library-and-Archive/ resource_documents/Resource-2014-Telepsychiatry-Clinical-Psychiatry.pdf (last accessed October 17, 2018).

9. ATA Telehealth Resource Toolbox. 2017. Available at www.americantelemed .org/main/policy-page/state-policy-resource-center/additional-state-resources (last accessed October 16, 2018).

10. Shore JH, Mishkind MC, Bernard J, Doarn CR, Bell Jr. I, Bhatia R, Brooks E, Caudill R, Cohn ER, Delphin BJ, Eppolito A, Fortney J, Friedl K, Hirsch P, Jordan PJ, Kim TJ, Luxton DD, Lynch MD, Maheu MM, McVeigh FL, Nelson E, Officer C, O'Neil PT, Roberts $\sqcup$, Rye C, Turvey C, Vo A. A lexicon of assessment and outcome measures for telemental health. Telemed J E Health 2014;20:282-292.

11. The American Telemedicine Association. Evidence-based practice for telemental health, 2009. Available at https://higherlogicdownload.s3.amazonaws.com/ AMERICANTELEMED/618da447-dee1-4ee1-b941- c5bf3db5669a/Uploadedlmages/ New\%20Guideline\%20Cover\%20May\%2017 /NEW ATA\%20Evidence\%2 OBased\%20Practice.pdf (last accessed October 16, 2018).

12. Grady B, Myers KM, Nelson EL, Belz N, Bennett L, Carnahan L, Rowe, N. Evidence-based practice for telemental health. Telemed J E Health 2011;17:131-148.

13. Hubley S, Lynch SB, Schneck C, Thomas M, Shore J. Review of key telemental health outcomes. World J Psychiatry 2016;6:269-282.

14. Bashshur RL, Shannon GW, Bashshur N, Yellowlees PM. The empirical evidence for telemedicine interventions in mental disorders. Telemed J E Health 2016; 22:87-113.

15. Hilty DM, Ferrer DC, Parish MB, Johnston B, Callahan EJ, Yellowlees PM. The effectiveness of telemental health. Telemed J E Health 2013;19:444-454.

16. Shore JH. Telepsychiatry: Videoconferencing in the delivery of psychiatric care. Am J Psychiatry 2013;170:256-262.

Address correspondence to: Jay H. Shore, MD, MPH

Department of Psychiatry

The University of Colorado Anschutz Medical Campus Aurora, CO 80045

E-mail: jay.shore@uchsc.edu

Received: September 13, 2018

Accepted: September 20, 2018 Online Publication Date: October 24, 2018 